\title{
Toxoplasmic Lymphadenopathy Presenting as a Palpable Axillary Mass - A Case Report
}

\author{
Gudrun Peters ${ }^{\mathrm{a}, \mathrm{c}}$, Catherine M Jones ${ }^{\mathrm{a}}$, Lynne Brothers ${ }^{\mathrm{b}}$
}

\begin{abstract}
Usually infection with Toxoplasma gondii is asymptomatic and no treatment is required. Less than $10 \%$ of the cases present with clinical symptoms such as lymphadenopathy in the head and neck region, asthenia, fever, cephalea and arthralgia. This case report illustrates an unusual case of Toxoplasmosis presenting as a palpable axillary mass in an otherwise healthy individual.
\end{abstract}

Keywords: Axillary adenopathy; Toxoplasmosis

\section{Introduction}

Infection with Toxoplasma gondii is usually asymptomatic in an immunocompetent host and no specific treatment is required. Less than $10 \%$ of infected subjects present with clinical symptoms [1]. Toxoplasmic lymphadenitis most commonly involves lymph nodes in the head and neck region [1, 2]. Leading clinical symptoms are non-specific and include asthenia, fever, cephalea and arthralgia [1]. Clinically significant extranodal disease is uncommon in immunocompetent individuals but myocarditis, pericarditis, myositis, pneumonitis, chorioretinitis and encephalitis can occur [2].

We present an unusual case of toxoplasmosis presenting as unilateral swelling of the axillary tail in an otherwise healthy individual.

Manuscript accepted for publication May 10, 2012

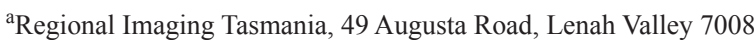
TAS, Australia

bWomen's Imaging, 18 Main Road, Moonah 7009 TAS, Australia

${ }^{\mathrm{c}}$ Corresponding author: Gudrun Peters, Regional Imaging Tasmania,

49 Augusta Road, Lenah Valley 7008 TAS, Australia.

Email: Gudrun.Peters@i-med.com.au

doi:10.4021/jmc692w

\section{Case Report}

A 47 year old woman presented to her general practitioner with a palpable right axillary mass. The patient also complained of mild tenderness over the mass. She had no history of malignancy, systemic disease or known recent viral illness. A previous mammogram from 6 months prior was normal.

Ultrasound of both axillae was performed. The left axilla was normal. The right axilla showed two significantly enlarged nodes, measuring $25 \times 16 \mathrm{~mm}$ and $24 \times 17 \mathrm{~mm}$. These nodes demonstrated asymmetrical cortical thickening with hyper vascularity and generalised hypoechoicity (Fig. 1-4).

A fine needle aspirate was subsequently performed. Cytology showed reactive lymphadenitis with follicular hyperplasia and non-necrotising granulomatosis. Toxoplasmosis was suggested as a possible aetiology and subsequently Toxoplasma serology was performed. Toxoplasma IgM antibodies were detected in keeping with a current or recent infection. As no other clinical symptoms occurred no specific treatment was initiated by the GP. A follow up serology test after 7 months was in keeping with past exposure to Toxoplasma.

\section{Discussion}

Isolated unilateral axillary lymphadenopathy is not an uncommon clinical symptom. A long list of differential diagnoses need to be considered. Axillary nodes drain the breast, thoracic wall and upper limb. Axillary adenopathy may be caused by local pathology such as infection, inflammation or malignancy in these regions. The most common cause of unilateral axillary adenopathy is upper limb infection or injury [3]. In female patients breast malignancy needs to be excluded. Systemic illness less commonly presents with unilateral axillary adenopathy. Hodgkin's and non-Hodgkin's lymphoma and other systemic malignancy such as melanoma metastases may rarely present in this way [3-5]. Autoimmune diseases such as rheumatoid arthritis, lupus, Sjogren's syndrome and dermatomyositis are further uncommon causes. 


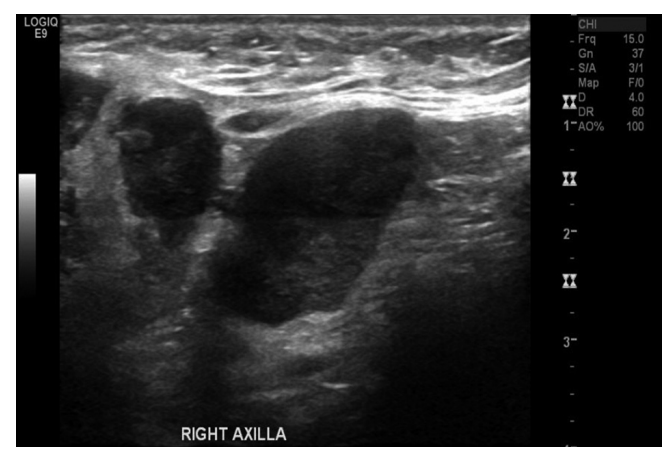

Figure 1. Ultrasound image of the right axilla showing enlarged lymph nodes with generalised hypoechoicity.

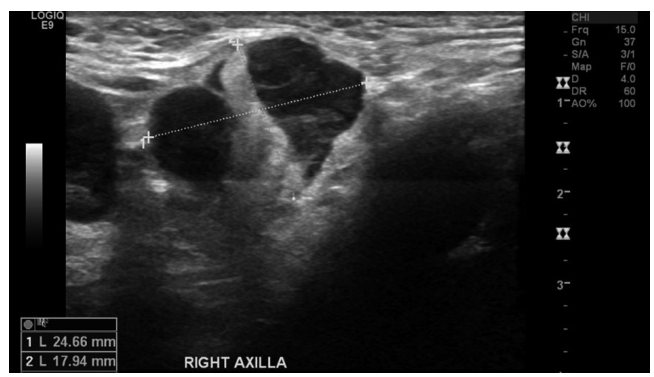

Figure 2. Ultrasound image of the right axilla showing enlarged lymph nodes with generalised hypoechoicity.

Other causes of axillary adenopathy include sarcoidosis and drug reaction $[3,6]$. Toxoplamosis presenting as unilateral axillary adenopathy is rare with only three case reports published to the knowledge of the authors [7-9].

Prevalence of toxoplasmosis is widespread and varies by age and geographic region, however less than $10 \%$ of the cases are symptomatic [1]. Most of these patients present with painless lymphadenopathy or asthenia [1]. Lymphadenitis with follicular hyperplasia and non-necrotising granulomatosis on cytology is suggestive of the disease, but correlation with serological titre levels is required for the diagnosis $[10,11]$. If fine needle aspirate cytology and serology suggest toxoplasmosis infection, no further invasive intervention is required for diagnosis $[10,11]$.

In this case, the diagnosis of toxoplasmosis was suggested from the fine needle aspirate cytology and serology confirmed a current or recent Toxoplasma infection and no further investigation or treatment was required.

\section{Conclusion}

Toxoplasma infection presenting as unilateral axillary adenopathy is rare. In cases where local infection or inflammation of the upper limbs, breast or thoracic wall is excluded, an FNA should be considered for further assessment. If the FNA result is suggestive of Toxoplasmosis then this should

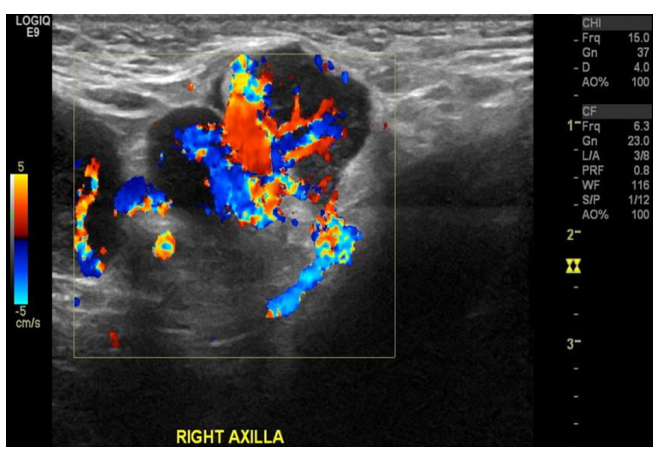

Figure 3. Enlarged axillary lymph nodes demonstrating hypervascularity on color Doppler flow investigation.

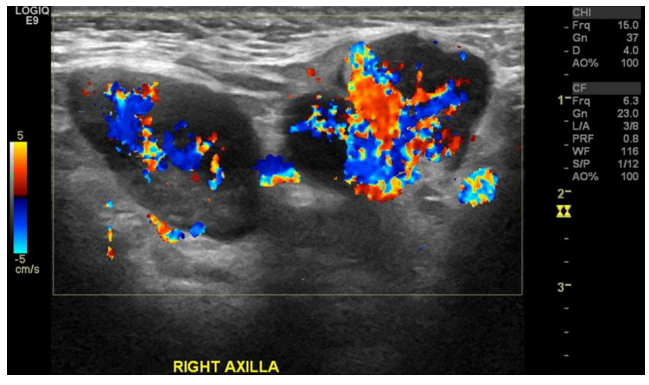

Figure 4. Enlarged axillary lymph nodes demonstrating hypervascularity on color Doppler flow investigation.

be confirmed serologically. FNA in conjunction with serology can avoid further more invasive interventions.

\section{Grant Support}

No financial or ethical disclosures.

\section{References}

1. Durlach RA, Kaufer F, Carral L, Hirt J. Toxoplasmic lymphadenitis--clinical and serologic profile. Clin Microbiol Infect. 2003;9(7):625-631.

2. McCabe RE, Brooks RG, Dorfman RF, Remington JS. Clinical spectrum in 107 cases of toxoplasmic lymphadenopathy. Rev Infect Dis. 1987;9(4):754-774.

3. Bazemore AW, Smucker DR. Lymphadenopathy and malignancy. Am Fam Physician. 2002;66(11):2103-2110.

4. Ferrer R. Lymphadenopathy: differential diagnosis and evaluation. Am Fam Physician. 1998;58(6):1313-1320.

5. Jose BO, Koerner P, Spanos WJ, Jr., Paris KJ, Silverman CL, Yashar C, Carrascosa LB. Hodgkin's lymphoma in adults--clinical features. J Ky Med Assoc. 2005;103(1):15-17.

6. Schwab FD, Burger H, Isenschmid M, Kuhn A, Mueller 
MD, Gunthert AR. Suspicious axillary lymph nodes in patients with unremarkable imaging of the breast. Eur $\mathrm{J}$ Obstet Gynecol Reprod Biol. 2010;150(1):88-91.

7. Siriwardana HP, Teare L, Kamel D, Inwang ER. Toxoplasmosis presenting as a swelling in the axillary tail of the breast and a palpable axillary lymph node mimicking malignancy: a case report. J Med Case Rep. 2011;5:348.

8. Yildirim AC, Bostanci H, Yilmaz ED, Kutluer N, Kargici $\mathrm{H}$. Toxoplasma lymphadenitis mimicking malign axillary lymphadenopathy of a left breast mass. Updates Surg. 2012.
9. Turner JR. Toxoplasmosis Presenting as a Swelling in the Axillary Tail of the Breast. Postgrad Med J. 1965;41:3940.

10. Viguer JM, Jimenez-Heffernan JA, Lopez-Ferrer P, Gonzalez-Peramato P, Vicandi B. Fine needle aspiration of toxoplasmic (Piringer-Kuchinka) lymphadenitis: a cytohistologic correlation study. Acta Cytol. 2005;49(2):139-143.

11. Gupta RK. Fine needle aspiration cytodiagnosis of toxoplasmic lymphadenitis. Acta Cytol. 1997;41(4):10311034. 